\title{
A comprehensive diagram to grow (0001)InGaN alloys by molecular beam epitaxy
}

\author{
Ž. Gačević , V.J. Gómez, N.García Lepetit, P.E.D. Soto Rodríguez, A. Bengoechea, S. Fernández-Garrido \\ R. Nötzel, E. Calleja
}

\begin{abstract}
A B S T R A C T
The composition, strain and surface morphology of (0001)InGaN layers are investigated as a function of growth temperature $\left(460-645^{\circ} \mathrm{C}\right)$ and impinging In flux. Three different growth regimes: nitrogen-rich, metal-rich and intermediate metal-rich, are clearly identified and found to be in correlation with surface morphology and strain relaxation. Best epilayers' quality is obtained when growing under intermediate metal-rich conditions, with 1-2 monolayers thick In ad-coverage. For a given In flux, the In incorporation decreases with increasing growth temperature due to $\mathrm{InN}$ thermal decomposition that follows an Arrhenius behavior with $1.84 \pm 0.12 \mathrm{eV}$ activation energy.
\end{abstract}

\section{Introduction}

The ternary $\operatorname{In}_{x} \mathrm{Ga}_{1-x} \mathrm{~N}$ alloys, with their band gaps (0.7-3.4 eV) covering the near infrared and the entire visible spectrum, have been receiving wide attention especially for optoelectronic device applications such as violet/blue laser and light emitting diodes (LEDs) [1]. Their employment for efficient green and red lighting still remains a challenge since a rapid decrease in InGaN quantum wells (QWs) performance is observed with increasing In content [2]. There are several properties, typical of (0001)InGaN QWs, that characterize and limit their optical performance: (i) a high dislocation density $\left(\sim 10^{9} \mathrm{~cm}^{-2}\right.$ ) that gives rise to non-radiative recombination; (ii) strong polarization effects that lead to electron-hole separation and a consequent decrease in radiative efficiency; and (iii) the composition/thickness fluctuations (especially for QWs with higher In content) that lead to exciton localization in regions with higher In content and/or well thickness. In the case of LEDs the localization is considered beneficial, since it keeps excitons away from dislocations preventing, thus, non-radiative recombination [3]. The situation is, however, very different for laser diodes (LDs), because the QWs composition/thickness fluctuations induce local band gap/confinement energy variations that affect the spectral purity of their emission. This is detrimental for LDs, since to sustain the lasing action, the optical gain has to surpass the high optical losses.

The progress in understanding the plasma-assisted molecular beam epitaxy (PA-MBE) growth mechanisms has led to recent demonstrations of both blue and green LDs [4-8], making this growth technique competitive to the traditionally used metal organic vapor phase epitaxy (MOVPE) $[1,9,10]$. A further advance, however, is conditioned by the material quality requirements, i.e. by flat and highly uniform InGaN layers with In contents above 20\%. Experimental evidence suggests that the best quality is obtained when growing under intermediate metal-rich conditions (i.e. with a selfregulating In ad-layer) at temperatures above $600{ }^{\circ} \mathrm{C}$ [11]. Due to the relatively weak bond, In-N dissociation starts around $500{ }^{\circ} \mathrm{C}$. Unlike other materials, InGaN is thus commonly grown at temperatures where strong material decomposition is present. Averbeck et al. proposed a semi-empirical model, estimating the InN thermal decomposition rate as proportional to the layer's In content and a Boltzmann factor with a corresponding activation energy $\left(\Phi_{\operatorname{lnN}}^{\mathrm{dec}}=C x_{\ln } e^{-E_{\mathrm{a}} / K T}\right)$ [12]. Interestingly, although the model has been experimentally verified by several groups, there is still a significant dispersion in the reported pre-factors and activation energies $[8,13,14]$. One reason is that the growth temperature range for InGaN is narrow $\left(450-650^{\circ} \mathrm{C}\right.$ ); i.e. $K T^{-1} \sim[12.5-16.0] \mathrm{eV}^{-1}$, making the experimental estimations prone to errors. In this paper, we give a detailed insight into how the growth parameters affect the InGaN composition, strain, and surface morphology. 


\section{Experimental}

The samples were grown in a RIBER Compact 21 MBE system equipped with a radio-frequency plasma nitrogen source and standard Knudsen cells for Ga and In. Commercial Lumilog (MOVPE grown) $\sim 4 \mu \mathrm{m}$ thick (0001)GaN-on-sapphire templates were used as substrates. Prior to InGaN growth, a $\sim 60 \mathrm{~nm}$ thick GaN buffer layer was grown under intermediate Ga-rich conditions, to bury possible impurities and to provide a flat surface [15]. The growth temperature $\left(T_{\mathrm{g}}\right)$ was measured using an Ircon Modline 3 optical pyrometer, calibrated by measuring the desorption rate of liquid In from the GaN surface [13]. Impinging metal fluxes of In ( $\left.\Phi_{\text {In }}\right)$ and Ga $\left(\Phi_{\mathrm{Ga}}\right)$ were measured in situ by a flux gage in equivalent pressure units (Torr) and calibrated in absolute units (atoms/ $\mathrm{cm}^{2} \mathrm{~s}$ ) making use of the thickness of $\mathrm{GaN}$ and $\mathrm{InN}$ samples grown under $\mathrm{N}$-rich conditions, measured by scanning electron microscopy (SEM) [16]. Similarly, the active nitrogen flux $\left(\Phi_{\mathrm{N}}\right)$ was calibrated making use of GaN samples grown under Ga-rich conditions. The InGaN growth time was set to $20 \mathrm{~min}$ for all samples, being thus the layer thickness $(60-120 \mathrm{~nm})$ proportional to the growth rate. The growth front was in situ monitored by reflection high-energy electron diffraction (RHEED) and the finishing growth surface was ex situ characterized by Nomarski optical microscopy and Digital Instruments MMAFM-2 atomic force microscopy (AFM). The growth rate, In content and strain were assessed by an X'Pert PRO PANalytical X-ray diffraction (XRD) system.

\section{Results}

The MBE growth conditions of InGaN are defined by four parameters: $T_{\mathrm{g}}, \Phi_{\mathrm{In}}, \Phi_{\mathrm{Ga}}$ and $\Phi_{\mathrm{N}}$, the growth problem being fourdimensional $\left(T_{\mathrm{g}}, \Phi_{\mathrm{In}}, \Phi_{\mathrm{Ga}}, \Phi_{\mathrm{N}}\right)$ by nature. To simplify it, we reduced its dimensionality to two by keeping $\Phi_{\mathrm{Ga}}$ and $\Phi_{\mathrm{N}}$ constant for all samples $\left(\Phi_{\mathrm{Ga}}=1.75 \times 10^{14}\right.$ atoms $/ \mathrm{cm}^{2} \mathrm{~s}, \Phi_{\mathrm{N}}=3.7 \times 10^{14}$ atoms $/$ $\mathrm{cm}^{2} \mathrm{~s}$ ). Two sample series were grown to investigate the influence of the remaining growth parameters $\left(T_{\mathrm{g}}, \Phi_{\mathrm{In}}\right)$ on the InGaN epilayer composition, strain and surface morphology.

For the growth temperature series $(\mathrm{A})$ the impinging In flux was set to $\Phi_{\mathrm{In}}=1.8 \times 10^{14}$ atoms $/ \mathrm{cm}^{2} \mathrm{~s}$ and the temperature was varied within the $T_{\mathrm{g}}=460-645^{\circ} \mathrm{C}$ range. The series was grown under nominally slightly nitrogen-rich (N-rich) conditions $\left(\Phi_{\mathrm{Ga}}+\Phi_{\mathrm{In}} \sim 0.96 \Phi_{\mathrm{N}}\right)$. Thus, no metal accumulation was observed below $510^{\circ} \mathrm{C}$ (confirmed by Nomarski) as the growth was carried out under nitrogen excess: $\Phi_{\mathrm{Ga}}+\Phi_{\mathrm{In}}<\Phi_{\mathrm{N}}$, yielding a bright spotty RHEED pattern. In the $510-600^{\circ} \mathrm{C}$ range, the RHEED pattern was in the form of diffuse slightly modulated streaks. In droplets were

a
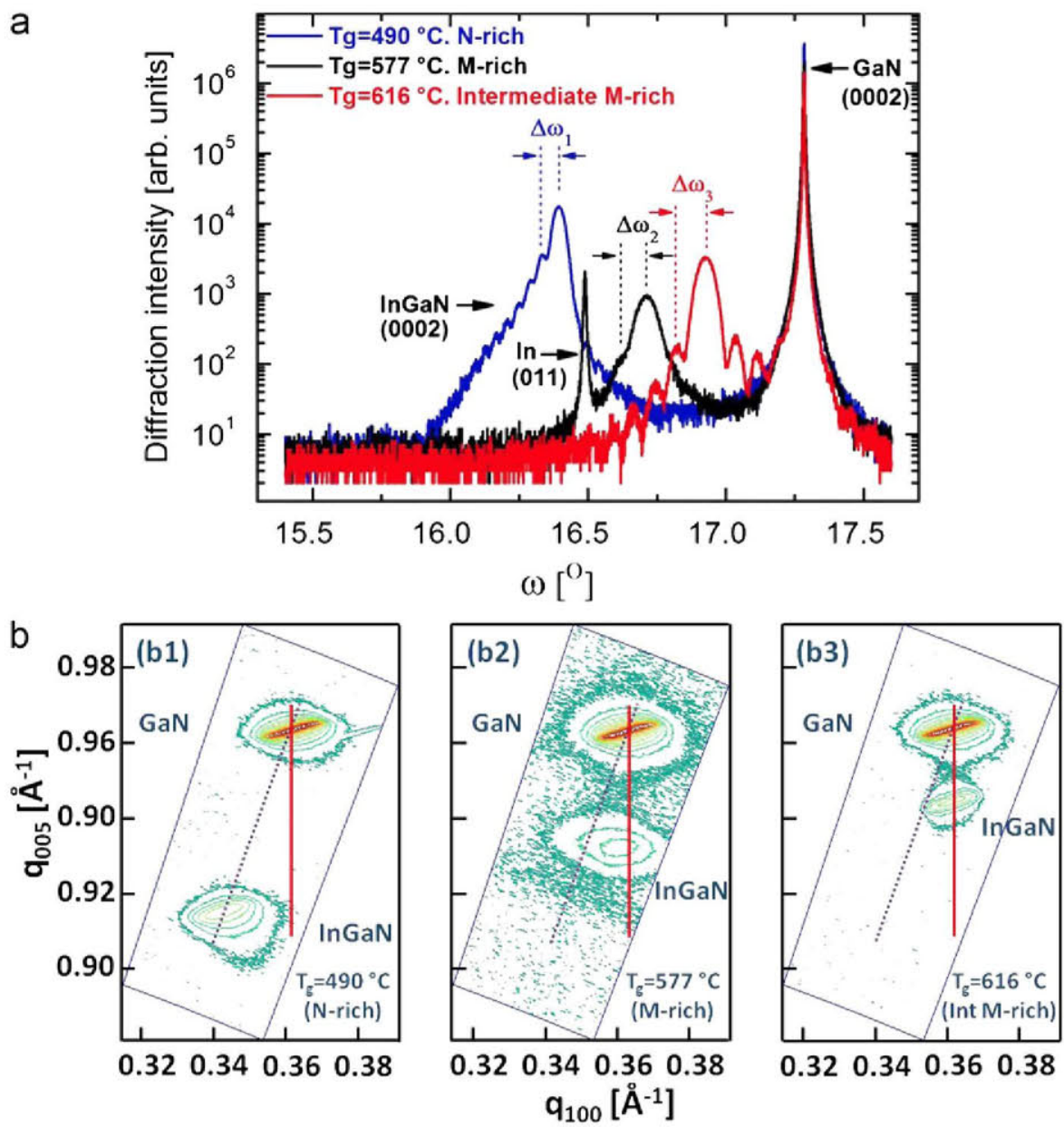

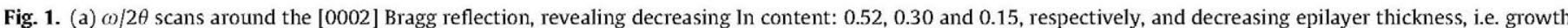

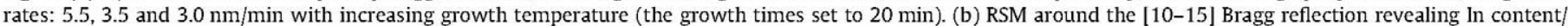

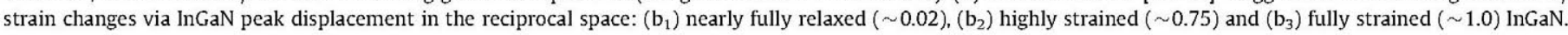

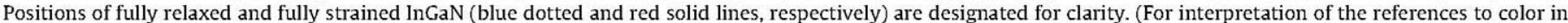
this figure legend, the reader is referred to the web version of this article.) 
observed upon the growth termination, confirming metal-rich (M-rich) growth conditions. In this temperature range, the additional In that is due to $\operatorname{lnN}$ thermal decomposition $\left(\Phi_{\operatorname{lnN}}^{\mathrm{dec}}\right)$ is higher than In losses due to In desorption ( $\left.\Phi_{\mathrm{In}}^{\mathrm{des}}\right)$, making the growth conditions effective by M-rich: $\Phi_{\mathrm{Ga}}+\Phi_{\mathrm{In}}+\Phi_{\mathrm{InN}}^{\mathrm{dec}}-$ $\Phi_{\mathrm{In}}^{\mathrm{des}}>\Phi_{\mathrm{N}}$. Once the temperature was increased above $600^{\circ} \mathrm{C}$, the RHEED pattern became sharp and perfectly streaky. Metal droplets disappeared as a consequence of In desorption: $\Phi_{\mathrm{Ga}}+\Phi_{\mathrm{In}}+\Phi_{\mathrm{InN}}^{\mathrm{dec}}-\Phi_{\mathrm{In}}^{\mathrm{des}} \leq \Phi_{\mathrm{N}}$. The growth was performed without metal accumulation on the growth front, i.e. with a stable In adcoverage, 0-2 monolayers (MLs) thick, under intermediate M-rich conditions [11,17].

The InGaN growth rate was assessed from the lateral fringes separation in XRD $\omega / 2 \theta$ scans around the [0002] Bragg reflection. The composition and normalized strain ( 0 for a fully relaxed and 1 for a fully strained epilayer) were estimated from $a$ and $c$ lattice parameters (determined by reciprocal space mapping (RSM) around the [10-15] Bragg reflection) making use of the linear III-nitride elasticity theory [18]. The increase in growth temperature thermally enhaces InN decomposition, leading to lower growth rates (the lateral fringes separation increases, Fig. 1a) and lower In contents (the InGaN peak shifts toward GaN, Fig. 1a, b).

AFM measurements confirmed that the samples' surface morphology is strongly influenced by the growth regime. Under $\mathrm{N}$ rich conditions, the initial InGaN nucleation occurs in the form of 3D islands (confirmed by spotty RHEED). The compact bulk layer is formed through coalescence of these initial nucleation seeds, whose diameter is typically in $20-100 \mathrm{~nm}$ range (Fig. 2a). The 3D growth brings high root mean square (RMS) surface roughness (RMS $>2 \mathrm{~nm}$ ), whereas the coalescence leads to early strain relaxation (Fig. 2a and e). Once the growth conditions shift to effective M-rich conditions, the nucleation occurs in the form of 2D layers (confirmed by streaky RHEED). The smoothest surface is obtained when the growth is performed under intermediate $\mathrm{M}$ rich conditions (RMS $\sim 1 \mathrm{~nm}$, Fig. $2 \mathrm{~b}$ and e), with a stable $1-2 \mathrm{MLs}$ In ad-layer excess on the growth front since the ad-coverage reduces the surface free energy and enhances the ad-atoms surface kinetics [11]. Further increase in temperature leads to In ad-coverage depletion ( $<1 \mathrm{ML}$ ), provoking significant surface deterioration (RMS $>2 \mathrm{~nm}$, Fig. 2c and e) since bare InGaN regions grow without In surfactant effect. Since increasing growth temperature leads to gradual decrease in In content and epilayer thickness, a gradual increase of residual strain can be expected with respect to it. Observe, however, the very sharp rise of residual strain (from $\sim 0.02$ to $\sim 0.50$ ) over the narrow temperature range $500-520^{\circ} \mathrm{C}$ (Fig. 2e). This rise is attributed to the growth regime transition from $\mathrm{N}$-rich to $\mathrm{M}$-rich conditions. $\mathrm{M}$-rich conditions improve wetting of the initial surface and lead to formation of bigger nucleation domains (Fig. 2a and b); thus lower density of defects is expected by the bigger domains' coalescence, postponing the early strain relaxation present under $\mathrm{N}$-rich conditions.

The $\mathrm{In} / \mathrm{Ga}$ incorporation rates $\left(\Phi_{\mathrm{In} / \mathrm{Ga}}^{\mathrm{inc}}\left[\right.\right.$ atoms $\left.\left./ \mathrm{cm}^{2} \mathrm{~s}\right]\right)$ were estimated by multiplying the In/Ga atoms surface density with the growth rate (assessed by XRD scans). Fig. 3a summarizes In and $\mathrm{Ga}$ incorporation rates as a function of the growth temperature (series A). As $\mathrm{Ga}$ incorporates preferentially to $\mathrm{In}$, and $T_{\mathrm{g}}$ remains far below the threshold temperature for GaN decomposition and/or $\mathrm{Ga}$ desorption (expected above $680^{\circ} \mathrm{C}$ ), Ga fully incorporates into the growing crystal. On the other hand, In fully incorporates only when growth is performed below $500{ }^{\circ} \mathrm{C}$ and under $\mathrm{N}$-rich conditions. At higher temperatures, In incorporation decreases monotonically with increasing growth temperature, due to thermal activation of InN decomposition. To determine the decomposition parameters $\left(C\right.$ and $E_{\mathrm{a}}$ ) according to the approximate model proposed by Averbeck and Riechert [12], we
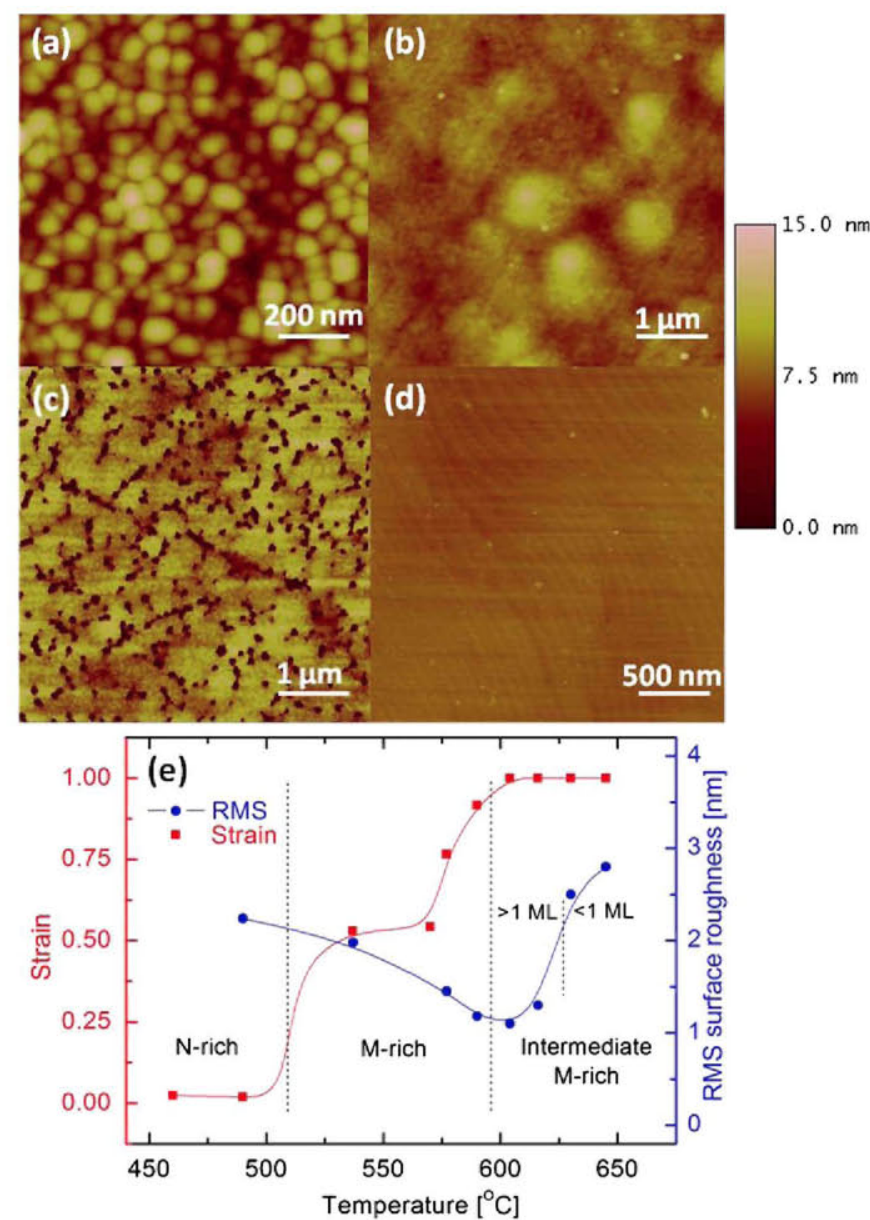

Fig. 2. AFM images of different InGaN surfaces: (a) N-rich, (b) intermediate M-rich, In ad-layer > $1 \mathrm{ML}$, (c) intermediate M-rich, In ad-layer < $1 \mathrm{ML}$ and (d) reference GaN. (e) Variation of in-plane strain (normalized) and RMS surface roughness as functions of growth regime and temperature. The solid lines are guides for the eye.

made use of estimated InN losses of the samples grown under Mrich or intermediate M-rich conditions with fully formed In ad-layer ( $>1 \mathrm{ML}$ ), Fig. 3c. The fitting yields an activation energy of $E_{\mathrm{a}}=1.84 \pm 0.12 \mathrm{eV}$ and a pre-exponential factor of $\ln (C)=58.5 \pm 1.6$, i.e. $C=2.55 \times 10^{25}$ atoms $/ \mathrm{cm}^{2} \mathrm{~s}$ (Fig. $3 \mathrm{~b}$ ).

The In content $\left(x_{\mathrm{In}}\right)$ under $\mathrm{N}$-rich conditions is determined by the metals impinging fluxes and In desorption:

$x_{\mathrm{ln}}=\left(\Phi_{\mathrm{ln}}-\Phi_{\mathrm{ln}}^{\mathrm{des}}\right) /\left(\Phi_{\mathrm{ln}}-\Phi_{\mathrm{ln}}^{\mathrm{des}}+\Phi_{\mathrm{Ga}}\right)$

whereas under M-rich conditions, the In content is determined by the Ga flux and available active nitrogen:

$x_{\mathrm{In}}=1-\Phi_{\mathrm{Ga}} /\left(\Phi_{\mathrm{N}}-\Phi_{\operatorname{lnN}}^{\mathrm{dec}}\right), \Phi_{\ln N}^{\mathrm{dec}}=C x_{\ln } e^{-E_{\mathrm{a}} / K T}$.

Notice that, under M-rich conditions, the In content can be determined as a function of growth temperature and impinging fluxes as follows:

$x_{\mathrm{In}}=\frac{\left(\Phi_{\mathrm{N}}+B\right)-\sqrt{\left(\Phi_{\mathrm{N}}+B\right)^{2}-4 B\left(\Phi_{\mathrm{N}}-\Phi_{\mathrm{Ga}}\right)}}{2 B}$

where $B=C e^{-E_{a} / K T}$ (see Eq. (2)).

The measured In content (Fig. 3c) is well fitted by Eq. (1), within the $\mathrm{N}$-rich regime $\left(\Phi_{\mathrm{In}}^{\mathrm{des}}=0\right.$ since $T_{\mathrm{g}}<500^{\circ} \mathrm{C}$ ), and by Eq. (3), within the M-rich and intermediate M-rich regimes, when the In ad-coverage is $>1 \mathrm{ML}$. When the In ad-coverage is partially depleted ( $<1 \mathrm{ML}$ ), Eq. (3) overestimates the actual In content. 

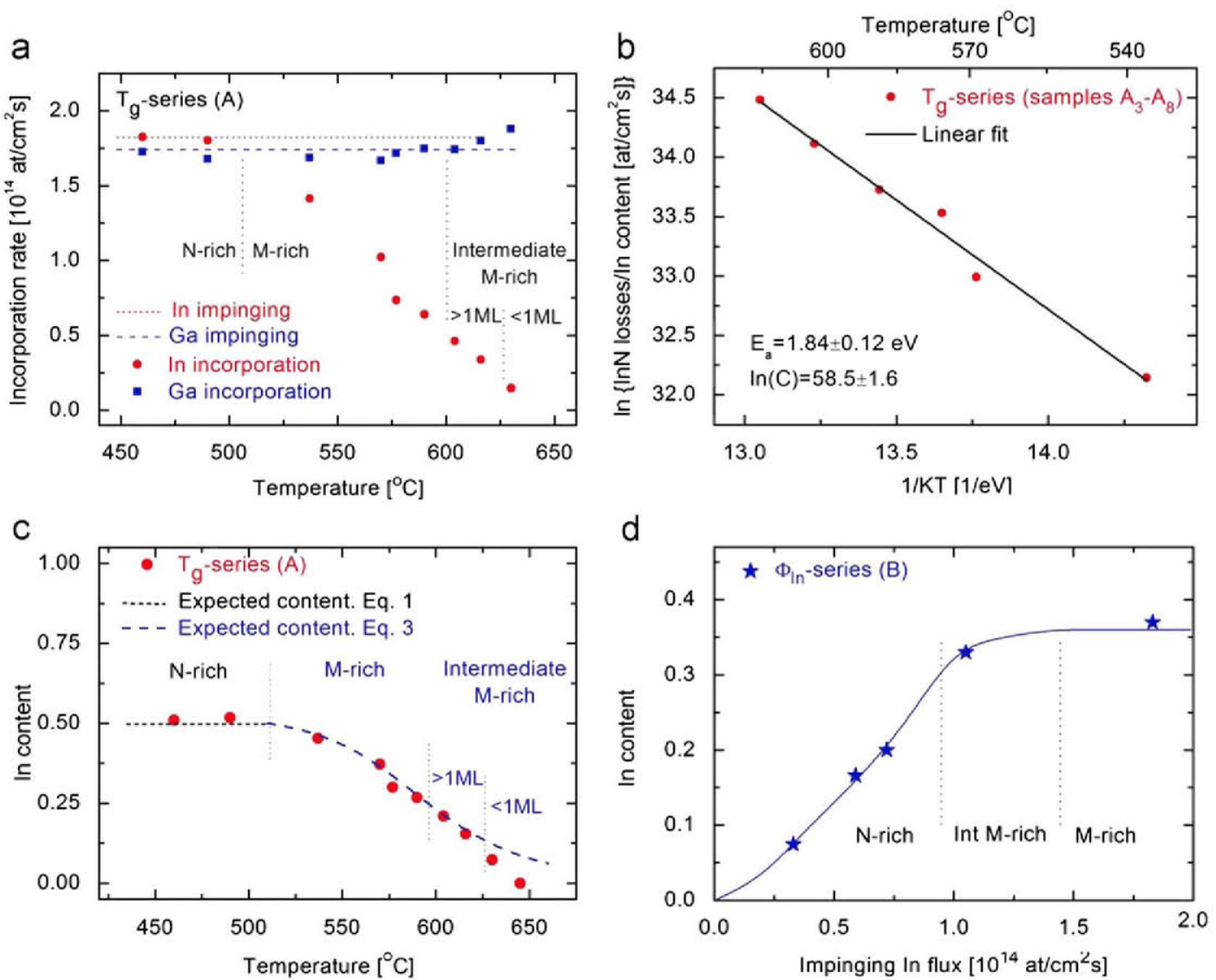

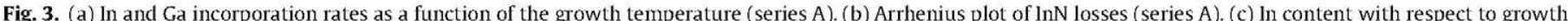

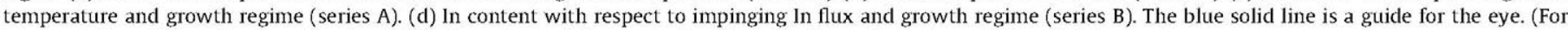
interpretation of the references to color in this figure legend, the reader is referred to the web version of this article.)

For the In impinging flux series (B), the temperature was set to $T_{\mathrm{g}}=570^{\circ} \mathrm{C}$ and the In impinging flux was varied within the $\Phi_{\text {In }}=0.3-1.8 \times 10^{14}$ atoms $/ \mathrm{cm}^{2} \mathrm{~s}$ range. Fig. $3 \mathrm{~d}$ summarizes the In content of the InGaN layer, as a function of impinging In flux. Under N-rich conditions, the increasing impinging In flux leads to increasing In incorporation, because there is active nitrogen available on the growth front. The relatively high growth temperature $\left(570^{\circ} \mathrm{C}\right)$ implies significant In desorption, the In content being determined by Eq. (1). Higher impinging In flux leads, first, to the formation of the In ad-layer (intermediate M-rich conditions) and then to the accumulation of In on the surface in the form of metal droplets (M-rich conditions). Note that once the In ad-layer is fully formed, a further increase in the impinging In flux brings no benefits to In incorporation, since there is no more active nitrogen available for the crystal growth. The In content saturates at the value determined by Eq. (2).

To unify all the results obtained, we constructed a comprehensive $2 \mathrm{D}\left(T_{\mathrm{g}}-\Phi_{\mathrm{In}}\right)$ InGaN growth diagram ( $\Phi_{\mathrm{Ga}}$ and $\Phi_{\mathrm{N}}$ fixed to $1.75 \times 10^{14}$ and $3.7 \times 10^{14}$ atoms $/ \mathrm{cm}^{2} \mathrm{~s}$, respectively). The effective stoichiometry determines the border of the effective $\mathrm{N}$-rich regime as a function of growth temperature: $\Phi_{\mathrm{In}}\left(T_{\mathrm{g}}\right)=\Phi_{\mathrm{N}^{-}} \Phi_{\mathrm{Ga}^{-}}$ $\Phi_{\operatorname{lnN}}^{\mathrm{dec}}\left(T_{\mathrm{g}}\right)$. Taking into account In desorption, the border between $\mathrm{M}$-rich and intermediate $\mathrm{M}$-rich conditions is determined as: $\Phi_{\mathrm{In}}\left(T_{\mathrm{g}}\right)=\Phi_{\mathrm{N}}-\Phi_{\mathrm{Ga}}-\Phi_{\operatorname{InN}}^{\mathrm{dec}}\left(T_{\mathrm{g}}\right)+\Phi_{\mathrm{In}}^{\mathrm{des}}\left(T_{\mathrm{g}}\right)$. Making use of the previously estimated InN thermal decomposition parameters and In desorption parameters reported by Gallinat et al. $\left(E_{\mathrm{a}}=2.49 \mathrm{eV}\right.$, $C=3.4 \times 10^{28}$ atoms $/ \mathrm{cm}^{2} \mathrm{~s}$ ) [13] the previous equations can be analytically solved, yielding the borders between the growth regimes (solid lines in Fig. 4). Observe, further, that according to our experimental evidence, the formation of full In ad-layer

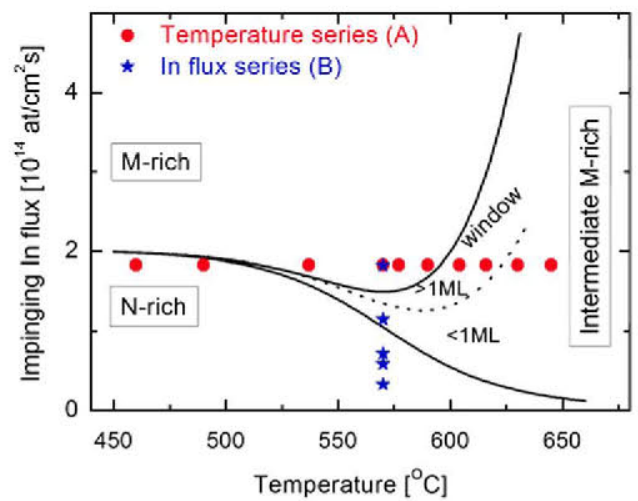

Fig. 4. InGaN growth diagram revealing different growth regimes as functions of growth temperature and impinging In flux $\left(\Phi_{\mathrm{N}}-\Phi_{\mathrm{Ca}}=1.95 \times 10^{14}\right.$ atoms $\left./ \mathrm{cm}^{2} \mathrm{~s}\right)$ The boundaries between the growth regimes (the solid lines) are determined analytically making use of $\operatorname{InN}$ decomposition and In desorption parameters (determined in this study and reported by Gallinat et al. [13], respectively).

(thickness between 1 and 2 MLs) provides smooth surface and stable conditions for InGaN growth (growth window, Fig. 4).

\section{Disscusion}

It is interesting to compare the results obtained for InN decomposition in this study with the previous ones reported for InN, InGaN and InAIN single layers. The first study of InN thermal decomposition, performed on InN samples, reported an $E_{\mathrm{a}}$ of $3.48 \mathrm{eV}$ [19]. 
Similar results, $3.45-3.85 \mathrm{eV}$, obtained for InGaN samples, were reported by Averbeck and Riechert, suggesting that $E_{a}$ may be a function of both In content and growth temperature [12]. Gallinat et al. studied the InN decomposition in situ by RHEED, and reported a value of $1.92 \pm 0.11 \mathrm{eV}$, relating it to the estimated $\mathrm{InN}$ bond energy, reported by Gallinat et al. [13] and Edgar [20]. Recently, Siekacz et al. studied InGaN samples and reported a slightly lower value, $1.6-1.7 \mathrm{eV}$ [8]. The value of $1.84 \pm 0.12 \mathrm{eV}$ obtained in this study is in good agreement with the results published by Gallinat et al. [13] and Siekacz et al. [8]. Observe that the value also compares well with our previous result obtained for InAlN single layers $\left(E_{\mathrm{a}}=2.0 \pm 0.2 \mathrm{eV}\right)$ [14]. We thus speculate that the dissociation of InN, for any of the three materials, can be well modeled with the activation energy corresponding to the native InN binary, although we cannot ignore that the presence of $\mathrm{Ga} / \mathrm{Al}$ atoms could slightly perturb this value. On the other hand, there is a huge difference in the pre-factors obtained for InGaN and InAlN $\left(\sim 2.55 \times 10^{25}\right.$ and $\sim 1.27 \times 10^{27}$ atoms $/ \mathrm{cm}^{2} \mathrm{~s}$, respectively), yielding a much higher InN dissociation rate in the latter case. We attribute this difference to a higher affinity of $\mathrm{Al}$ atoms toward $\mathrm{N}$, and, thus, higher probability of In-N bond breakage in the presence of Al than $\mathrm{Ga}$ atoms. This explains why it is so difficult to incorporate $\sim 18 \%$ of In into InAlN, under intermediate M-rich conditions (to obtain layers in-plane lattice-matched to $\mathrm{GaN}$ ) although the same molar fraction is easily incorporated into InGaN [14,21-23].

\section{Summary}

We have investigated the composition, strain and surface morphology of (0001)InGaN single layers as a function of growth temperature and impinging In flux, and constructed a comprehensive InGaN growth diagram that distinguishes three growth regimes: $\mathrm{N}$-rich, M-rich and intermediate M-rich. We observed a strong correlation between growth regime and the strain relaxation degree; $\mathrm{N}$-rich conditions favor the growth of relaxed layers whereas (intermediate) M-rich conditions favor the growth of strained layers. Within the intermediate M-rich regime, we show that the smoothest surface is obtained when the growth is performed with a full In adlayer ( $>1 \mathrm{ML}$ ) on the growth front. The InN losses resulting from InN thermal decomposition follow an Arrhenius behavior (activation energy of $1.84 \pm 0.12 \mathrm{eV}$ ) in agreement with recently published values for InN, InGaN and InAlN. The strong difference in the pre-factors, observed for InGaN and InAlN ternaries has been attributed to the lower Ga affinity toward nitrogen and linked to a lower InN dissociation rate of the former compound.

\section{Acknowledgments}

This work was partially supported by research grants from the Spanish Ministry of Education under Grant nos. MAT2008-04815 and Consolider CSD2006-19 and the Community of Madrid under Grant no. P2009/ESP-1503.

\section{References}

[1] S. Nakamura, S.J. Pearton, G. Fasol, The Blue Laser Diode: The Complete Story, 2nd ed., Springer-Verlag, 2000.

[2] Y.H. Cho, S.K. Lee, H.S. Kwack, J.Y. Kim, K.S. Lim, H.M. Kim, T.W. Kang, S.N. Lee, M.S. Seon, O.H. Nam, Y.J. Park, Applied Physics Letters 83 (2003) 2578.

[3] Ž. Gačević, A. Das, J. Teubert, Y. Kotsar, P.K. Kandaswamy, Th. Kehagias, T. Koukoula, Ph. Komninou, E. Monroy, Journal of Applied Physics 109 (2011) 103501.

[4] S. Hooper, M. Kauer, V. Bousquet, K. Johnson, J. Barnes, J. Heffernan, Electronics Letters 40 (1) (2004) 33.

[5] S.E. Hooper, M. Kauer, V. Bousquet, K. Johnson, C. Zellweger, J. Heffernan, Journal of Crystal Growth 278 (2005) 361.

[6] C. Skierbiszewski, Z. Wasilewski, M. Siekacz, A. Feduniewicz, P. Perlin, P. Wisniewski, J. Borysiuk, I. Grzegory, M. Leszczynski, T. Suski, S. Porowski, Applied Physics Letters 86 (2005) 011114.

[7] C. Skierbiszewski, P. Wisniewski, M. Siekacz, P. Perlin, A. Feduniewicz-Zmuda, G. Nowak, I. Grzegory, M. Leszczynski, S. Porowski, Applied Physics Letters 88 (2006) 221108

[8] M. Siekacz, M. Sawicka, H. Turski, G. Cywinski, A. Khachapuridze, P. Perlin, T. Suski, M. Bockowski, J. Smalc-Koziorowska, M. Krysko, R. Kudrawiec, M. Syperek, J. Misiewicz, Z. Wasilewski, S. Porowski, C. Skierbiszewski, Journal of Applied Physics 110 (2011) 063110.

[9] T. Miyoshi, S. Masui, T. Okada, T. Yanamoto, T. Kozaki, S. Nagahama, T. Mukai, Applied Physics Express 2 (2009) 062201.

[10] D. Queren, A. Avramescu, G. Brüderl, A. Breidenassel, M. Schillgalies, S. Lutgen, U. Strauß, Applied Physics Letters 94 (2009) 081119.

[11] E. Monroy, B. Daudin, E. Bellet-Amalric, N. Cogneau, D. Jalabert, F. Enjalbert, J. Brault, J. Barjon, L.S. Dang, Journal of Applied Physics 93 (2003) 1550.

[12] R. Averbeck, H. Riechert, Physica Status Solidi A 176 (1999) 301.

[13] C.S. Gallinat, G. Koblmüller, J.S. Brown, J.S. Speck, Journal of Applied Physics 102 (2007) 064907.

[14] S. Fernández-Garrido, Ž. Gačević, E. Calleja, Applied Physics Letters 93 (2008) 191907.

[15] G. Koblmüller, S. Fernandez-Garrido, E. Calleja, J.S. Speck, Applied Physics Letters 91 (2007) 161904

[16] B. Heying, R. Averbeck, L.F. Chen, E. Haus, H. Reichert, J.S. Speck, Journal of Applied Physics 88 (2000) 1855.

[17] S. Choi, T.H. Kim, S. Wolter, A. Brown, H.O. Everitt, M. Losurdo, G. Bruno, Physical Review B 77 (2008) 115435.

[18] I. Vurgaftman, J.R. Meyer, Journal of Applied Physics 94 (2003) 3675.

[19] O. Ambacher, M.S. Brandt, R. Dimitrov, T. Metzger, M. Stutzmann, R.A. Fischer, A. Miehr, A. Bergmaier, G. Dollinger, Journal of Vacuum Science and Technology B 14 (1996) 3532.

[20] J.H. Edgar, Group III Nitrides, INSPEC, London, 1994.

[21] Ž. Gačević, S. Fernández-Garrido, E. Calleja, E. Luna, A. Trampert, Physica Status Solidi C 6 (2009) S643.

[22] Ž. Gačević, S. Fernández-Garrido, D. Hosseini, S. Estradé, F. Peiró, E. Calleja, Journal of Applied Physics 108 (2010) 113117.

[23] Ž. Gačević, S. Fernández-Garrido, J.M. Rebled, S. Estradé, F. Peiró, E. Calleja, Applied Physics Letters 99 (2011) 031103. 